
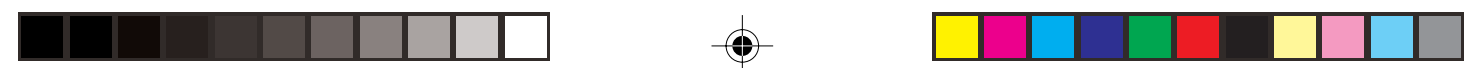

\title{
DIVERSIDAD Y ABUNDANCIA DE MURCIÉLAGOS EN SELVAS SECAS DE ESTACIONALIDAD CONTRASTANTE EN EL OESTE DE MÉXICO
}

\author{
CUAUHTÉMOC CHÁVEZ Y GERARDO CEBALLOS
}

Instituto de Ecología, Universidad Nacional Autónoma de México, Apartado 70-275, México, D. F. 04510, MÉXICO.

Resumen. Las selvas bajas y medianas tropicales del oeste de México presentan características estructurales y fenológicas contrastantes, que se traducen en diferencias notables en la diversidad de especies de muchos grupos de plantas y animales. A pesar de que los murciélagos juegan un papel muy importante en la estructura y función de esas selvas se desconoce como varía su diversidad en tales ecosistemas. En este estudio examinamos los patrones de riqueza y abundancia de los murciélagos en una selva baja y una selva mediana en la Reserva de la Biosfera Chamela - Cuixmala, Jalisco, México. En el periodo de febrero de 1992 a enero de 1994 muestreamos 18 meses, 36 noches y 180 horas, en un esfuerzo equivalente a 16,416 m² de red para cada ambiente. En total capturamos a 338 ejemplares de 15 especies. Nuestra predicción de encontrar diferencias importantes en la estructura y diversidad de la comunidad de murciélagos en ambos ambientes fue apoyada por la información recabada. El hábitat con mayor riqueza específica fue la selva baja con 13 especies; en la selva mediana registramos solo 10 especies. El índice de diversidad fue de H'= 1.99 y H’=1.40 para la selva baja y la selva mediana, respectivamente. El mayor número de individuos capturados fue en la selva baja. Las especies que capturamos únicamente en la selva baja fueron Carollia subrufa, Leptonycteris curasoae, Musonycteris harrisoni, Pteronotus davyi y P. parnelli, mientras que, en la selva mediana fue Rhogeessa parvula. Las especies más abundantes en los la selva baja fueron Artibeus jamaicensis, A. intermedius, Glossophaga soricina y Dermanura phaeotis, mientras que en la selva mediana sólo A. jamicensis y A. intermedius fueron abundantes.

Abstract. Tropical dry and semi-dry forests of western Mexico show contrasting structural and phenologic characteristics that result in important differences in the species diversity of many groups of plants and animals. Despite the importance of bats in the structure and function of these forests, the variation in diversity in these ecosystems is not known. This study examines the patterns of richness and abundance of bats in a tropical dry and tropical semi-dry forest in the Biosphere Reserve of Chamela - Cuixmala, Jalisco, Mexico. Between Februray 1992 and January 1994 we sampled 18 months, 36 nights and 180 hours, in an equivalent effort of 16,416 $\mathrm{m}^{2}$ of mist-nets for each environment. In total, we captured 338 individuals of 15 species. Our prediction of finding important differences in the structure and diversity of the bat community in both environments was supported by our findings. The habitat with highest specific richness was the dry forest, with 13 species; in the semi-dry forest we only recorded 10 

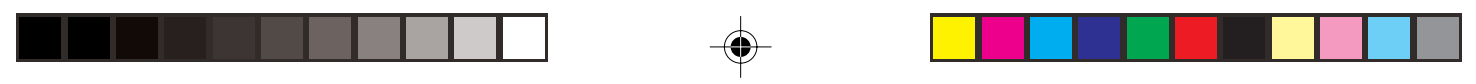

species. The diversity index was respectively $\mathrm{H}^{\prime}=1.99$ and $\mathrm{H}^{\prime}=1.40$ for the dry and semi-dry forest. The greatest number of individuals was captured in the dry forest. The species captured solely in the dry forest were Carollia subrufa, Leptonycteris curasoae, Musonycteris harrisoni, Pteronotus davyi and P. parnelli, while in the semi-dry forest was Rhogeessa parvula. The species more abundant in the dry forest were Artibeus jamaicensis, A. intermedius, Glossophaga soricina and Dermanura phaeotis, while in the semi-dry forest only A. jamicensis and $A$. intermedius were abundant.

Palabras clave: Diversidad, abundancia, murciélagos, temporalidad, selvas secas, selva baja, selva mediana, Jalisco, México.

\section{INTRODUCCIÓN}

Las selvas bajas y medianas del Pacífico americano, conocidas genéricamente como selvas secas, se encuentran entre los ecosistemas más amenazados por la deforestación masiva (Ceballos y García, 1995; Janzen, 1988). Estas selvas se caracterizan por una marcada estacionalidad en la temporada de lluvias, con una prolongada sequía. Los efectos de la estacionalidad climática son enormes y determinan en gran parte los ciclos estacionales y anuales en la fenología de las plantas (Bullock et al., 1995), que a su vez afectan la condiciones microclimáticas y la disponibilidad de alimento para las poblaciones de animales (e.g. Ceballos, 1995).

Las especies animales han desarrollado mecanismos conductuales, fisiológicos o ecológicos diversos para enfrentar la estacionalidad climática, entre los que encuentran movimientos a escalas regionales o biogeográficas, cambios en los patrones de actividad diaria y estacional, adaptaciones fisiológicas para enfrentar la escasez de agua, cambios en la dieta, acumulación de grasa o semillas (e.g., Janzen, 1983; Ceballos, 1995).

Muchas especies de vertebrados tienen la movilidad para desplazarse desde pocos metros hasta miles de kilómetros para evitar las épocas de escasez de agua o alimento (Fleming et al., 1993; Janzen, 1983). Por ejemplo, las poblaciones del murciélago magueyero (Leptonycteris curasoae) se desplazan de la región de Chamela - Cuixmala en la costa de Jalisco hasta Baja California, Sonora y Arizona para reproducirse (Ceballos et al., 1997). Otras especies como Diclidurus albus o Natalus stramineus están presentes únicamente durante el invierno o el verano, respectivamente, sin que se conozca a donde se desplazan durante otras épocas del año (Ceballos y Miranda, 1986; Sánchez Hernández y Chávez, 1985). Finalmente, existen evidencias diversas de que hay especies que usan habitats diferencialmente (Fleming, 1988; Rodríguez-H. y La Val, 2002; Stoner, 2001).

Los movimientos de las especies se reflejan en cambios temporales y espaciales en la estructura y diversidad de las comunidades de murciélagos de las selvas secas (Fleming et al., 1972; Hetihaus et al., 1975; La Val y Fitch, 1977; Stoner, 2001). 
Existen, sin embargo, muy pocos estudios que hayan evaluado este fenómeno en las selvas secas del oeste de México (Ceballos y Miranda, 1986, 2000; Chávez, 1996; Ramírez Pulido y Armella, 1987). Por lo tanto el objetivo principal de este estudio es determinar la variación temporal en la diversidad, la riqueza y la abundancia de las especies de murciélagos en dos selvas estacionales con fenología contrastante.

\section{MÉTODOS}

\section{Área de Estudio}

Los murciélagos fueron capturados en la Reserva de la Biosfera Chamela-Cuixmala (RBCCH), localizada en la costa de Jalisco, en el oeste de México. La reserva comprende alrededor de 13,000 hectáreas, que protegen selvas estacionales, manglares y otros ambientes (Ceballos y Miranda, 1986, 2000). El clima de la región pertenece a los cálido-húmedos, con una temperatura media anual de $24.9^{\circ} \mathrm{C}$, con un intervalo entre 29 y $32{ }^{\circ} \mathrm{C}$ como máxima y $18{ }^{\circ} \mathrm{C}$ como mínima. Entre las características sobresalientes de este clima son la concentración de las lluvias entre los meses de julio a octubre, y un prolongado periodo de sequía desde noviembre hasta mayo. El promedio de precipitación es de 748 mm/año (Bullock, 1986).

Los murciélagos se capturaron en dos selvas de características contrastantes denominadas selva baja y selva mediana, respectivamente. Estas selvas presentan un gran contraste en estructura y fenología. La selva mediana es más alta (hasta 25 m de altura), con una mayor diversidad de especies de árboles y bejucos, y una mayor productividad primaria (Ceballos, 1989; Martínez Yriazar, 1995). La selva baja se caracteriza por la baja altura de las especies arbóreas ( 5 a $10 \mathrm{~m}$ de altura) y porque la mayoría de las especies pierden sus hojas por un periodo de 5 a 7 meses del año, provocando esto un enorme contraste fisonómico entre la época de secas y de lluvias. Las especies de árboles más abundantes son Croton pseudoniveus, Heliocarpus pallidus, Lonchocarpus constrictus, Cordia allidora, Cordia eleagnoides, Caesalpinia eriostachys y Trichilia trifolia (Lott et al., 1987). La selva mediana en la RBCCH se encuentra restringida a los cauces de los arroyos y su extensión es muy limitada. Sus características fisonómicas presentan dos estratos arbóreos bien definidos, uno de hasta $15 \mathrm{~m}$ de altura y otro de $16-25 \mathrm{~m}$ y sólo de un 50 a $75 \%$ de las especies pierden sus hojas en la época de sequía. Los árboles dominantes son Thouinidium decandrum, Trichilia trifolia, Recchia mexicana, Ficcus spp, Foresteronia spicata, Capparis verrucosa, y Paulinia sessiflora (Lott et al., 1987). La separación entre los sitios de muestreo fue de $4.6 \mathrm{~km}$ en línea recta. El sitio de muestreo en la selva baja (SB) se ubicó a un costado de un camino en la reserva (19'23'46'” N y 104'59'34' 'W). El sitio de muestreo en la selva mediana (SM) se localizó a un costado del río Cuixmala (19²5’01'’ $\mathrm{N}$ y 10457’31'’ W ). 
Capturamos a los murciélagos en los dos sitios por medio de 3 redes de nylon (38 m lineales, $91 \mathrm{~m}^{2}$ ), que colocamos hasta $3 \mathrm{~m}$ de altura dentro de la vegetación. Aunque distintos autores mencionan la importancia de situar las áreas de muestreo de murciélagos en las cercanías a un cuerpo de agua, nosotros decidimos poner los sitios de muestreo en lugares bien conservados y representativos de cada selva, para evitar sesgos (Iudica, 1994). Debido al método de muestreo, los ensamblajes muestreados se limitan a especies que tienen una actividad en el sotobosque (Francis, 1990).

Llevamos a cabo muestreos mensuales entre marzo de 1992 y febrero de 1994, colocando las redes dos días durante la luna nueva, ya que la actividad de los murciélagos es marcadamente inhibida por la luna llena (Erkert, 1982). Identificamos a todos los ejemplares capturados apoyados en las claves de Ceballos y Miranda, 1986). Para cada ejemplar registramos la especie, hora de captura, sexo, peso, longitud del antebrazo, clase de edad y estado reproductivo en hembras (i.e. estado de las glándulas mamarías: no visibles, largas o lactantes) y machos (i.e. posición de los testículos: escrotados o abdominales). A cada murciélago capturado se le colocó una marca en el cuello con un código numérico por colores (Amín y Medellín, 1993).

Para determinar si la riqueza especifica y la abundancia difirieron con base al esfuerzo de muestreo utilizamos modelos log lineales (GLIM version 3.77). Para el análisis de los datos construimos tablas de contingencia de tres dimensiones, para conocer las relaciones entre las variables observadas en cuanto al número de especies capturadas por sitio, por hora y por temporada o mes. Adicionalmente calculamos un índice de abundancia relativa mensual por tipo de selva (Medellín, 1993). Como este índice sólo indica el número de individuos por metro red/hora y no da información por unidad de área, hicimos una ligera modificación multiplicando el ancho por el largo de la red, lo cual estimo el número de individuos por metro cuadrado de red por hora (individuos $/ \mathrm{m}^{2}$ de red). La diferencia con la estimación de Medellín (1993) es que él estimó metros lineales de redes sin considerar una unidad de área. Este índice lo utilizamos para comparar la abundancia relativa de los murciélagos más abundantes entre temporadas y años, utilizando una Anova paramétrica ó una Anova no parámetrica de Kurskal-Wallis dependiendo si nuestros datos cumplían los supuestos del análisis (Sigma Stat vers. 2.03).

Además elaboramos curvas de acumulación de especies. Para estimar su representatividad sobre la comunidad aplicamos una ecuación asintótica basada en cuatro modelos parámetricos; tres de ellos (i.e. Clench, Bertalanffy y Logarítmico) han sido ampliamente utilizados (Soberón y Llorente, 1993) y el multinomial (Nakamura y Peraza, 1995) estima la probabilidad de captura de los murciélagos. Nosotros utilizamos los siguientes criterios para seleccionar cual de los modelos utilizados se ajustó mejor a los datos: el coeficiente de correlación (r), el porcentaje de varianza (var), la asíntota y el número de residuos estandarizados (res) que se salen del intervalo -2 a 2 (Chávez, 1996). 
En relación a su abundancia relativa clasificamos a las especies como abundantes si se capturaron más de 30 individuos, comunes si eran de 15 a 29 ejemplares, y escasas si con capturas menores de 14 individuos.

\section{RESULTADOS}

\section{Base de datos y composición de especies}

Se muestrearon un total de 37,392 $\mathrm{m}^{2}$ de red que corresponden a 410 horas en 82 noches. Los datos que se utilizaron para las comparaciones corresponden a 18 meses, 36 noches, 180 horas de captura, con un total de 16,416 $\mathrm{m}^{2}$ de red en total para cada uno de los dos sitios; i.e. la selva baja (SB) y la selva mediana (SM).

Se capturaron un total de 338 individuos y 15 especies que corresponden al 42 \% del total de especies registradas para el área. La mayoría (13) de las especies fueron de la familia Phyllostomidae (Micronycteris microtis, Desmodus rotundus, Artibeus jamaicensis, A. intermedius, Dermanura phaeotis, Carollia subrufa, Centurio senex, Glossophaga soricina, Leptonycteris curasoae, Sturnira lilium y Musonycteris harrisoni); 3 especies pertenecieron a la familia Mormoopidae (Mormoops megallophyla, Pteronotus davyi y P. parnelli) y 1 especie la familia Vespertilionidae (Rhogeessa parvula).

\section{Diversidad y composición de especies}

La riqueza de la selva baja fue de 13 especies, de las cuales las siguientes no fueron capturadas en la selva mediana: C. subrufa, L. curasoae, M. harrisoni, P. davyi, y P. parnelli. Por otro lado, en la selva mediana se capturaron 10 especies, de las que fueron exclusivas $M$. microtis y $R$. parvula (Cuadro 1; Figura 1). Las 8 especies comunes entre los dos sitios fueron $M$. megallophyla, D. rotundus, A. jamaicensis, $A$. intermedius, D. phaeotis, C. senex, G. soricina y S. lilium. La diversidad de la selva baja $\left(H^{\prime}=1.99\right)$ fue significativamente mayor que la de la selva mediana $\left(H^{\prime}=1.40\right.$; $\mathrm{t}=13.07, \mathrm{p}<0.01, \mathrm{n}=18 \mathrm{~V}=250$ ).

El número acumulado de especies como una función del esfuerzo de captura fue muy similar para ambas selvas (Figura 2 a y b). Como se puede apreciar en todos los modelos evaluados casi se llegó a una asíntota, que indicó que las comunidades fueron bien muestreadas (Cuadro 2). Los modelos utilizados estimaron que en la comunidad de la selva baja se podrían esperar de 15 a 18 especies, y que el modelo que dio el mejor ajuste (Clench) indicó 18 especies. Para la selva mediana la estimación fue de 10 a 13 especies y el modelo que mejor se ajustó fue el Multinomial. 
vol. 5

Cuadro 2. Resultados de los ajustes de los diferentes modelos de acumulación de especies con diferentes intensidades de muestreo en la Reserva de la Biosfera Chamela-Cuixmala, México. $\mathrm{SB}=$ selva baja y $\mathrm{SM}=$ selva mediana. $\mathrm{sp}$ = especies, var = prcentaje de varianza, $\mathrm{r}$ = coeficiente de correlación y res = número de residuos estandarizados.

\begin{tabular}{|c|c|c|c|c|c|c|c|}
\hline \multicolumn{8}{|c|}{ Clench } \\
\hline & sp & var & $\mathrm{r}$ & a & $\mathrm{b}$ & $\mathrm{a} / \mathrm{b}$ & res \\
\hline SB & 13 & 91 & 0.95 & 0.79 & 0.05 & 18 & 0 \\
\hline SM & 10 & 90 & 0.95 & 0.04 & 0.03 & 13 & 5 \\
\hline \multicolumn{8}{|c|}{ Bertalanffy } \\
\hline & $\mathrm{sp}$ & var & $r$ & a & $\mathrm{b}$ & $\mathrm{a} / \mathrm{b}$ & res \\
\hline SB & 13 & 89 & 0.95 & 0.58 & 0.04 & 14.5 & 3 \\
\hline SM & 10 & 89 & 0.95 & 0.33 & 0.03 & 10.3 & 4 \\
\hline \multicolumn{8}{|c|}{ Logarítmico } \\
\hline & $\mathrm{sp}$ & var & $r$ & $\mathrm{a}$ & $\mathrm{z}$ & & res \\
\hline SB & 13 & 92 & 0.95 & 1.33 & 0.23 & & 4 \\
\hline SM & 10 & 90 & 0.95 & 0.50 & 0.25 & & 6 \\
\hline \multicolumn{8}{|c|}{ Multinomial } \\
\hline & $\mathrm{sp}$ & & $\mathrm{r}$ & $\mathrm{a}$ & $\mathrm{b}$ & k & res \\
\hline SB & 13 & & 93 & 1.14 & 12 & 15 & 0 \\
\hline SM & 10 & & 94 & 1.64 & 30 & 10 & 2 \\
\hline
\end{tabular}




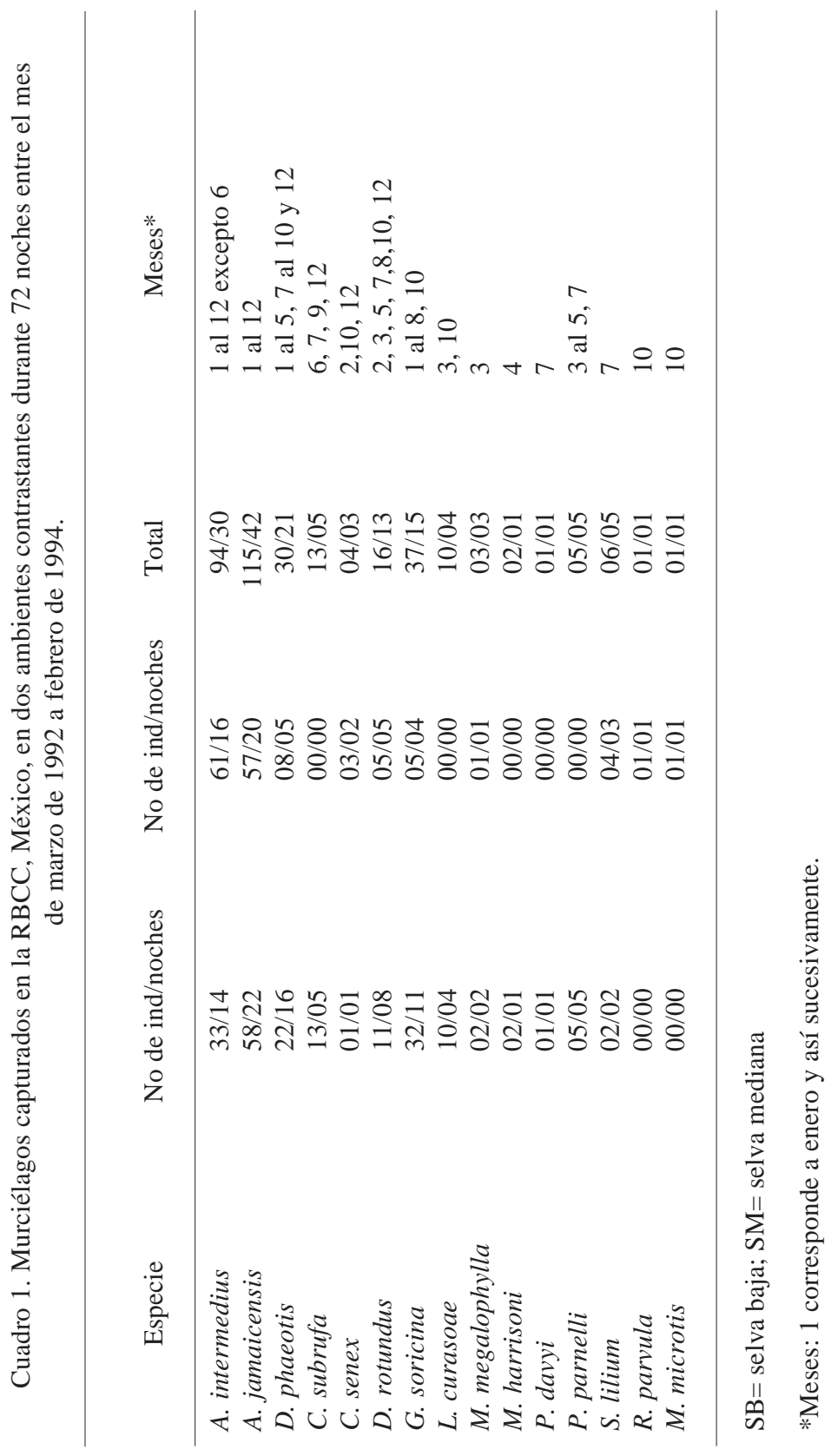




\section{Abundancias relativas}

Las especies se clasificaron como abundantes si estaban representadas por más de 21 individuos, comunes si tenían de 8 a 20 y escasas si se capturaron menos de 7 individuos. Los murciélagos más abundantes fueron frugívoros (293 individuos, 87\%) representados por 6 especies. Las tres especies más abundantes fueron $A$. jamaicensis, A. intermedius y D. phaeotis, con 239 individuos que representaron el $71 \%$ del número total de capturas durante todo el período de muestreo. Estas tres especies fueron dominantes y proveyeron la base para la diversidad de la comunidad y sus cambios alrededor del año.

En la selva baja se registraron 191 individuos de las 13 especies. Artibeus jamaicensis, A. intermedius, G. soricina y D. phaeotis fueron abundantes; C. subrufa, $D$. rotundus y $L$. curasoae fueron comunes, y las seis especies restantes fueron escasas. En la selva mediana se capturaron 146 individuos de 10 especies, de las cuales A. intermedius y A. jamaicensis fueron abundantes, D. phaeotis fue común, y G. soricina y las seis especies restantes fueron escasas (Figura 1).

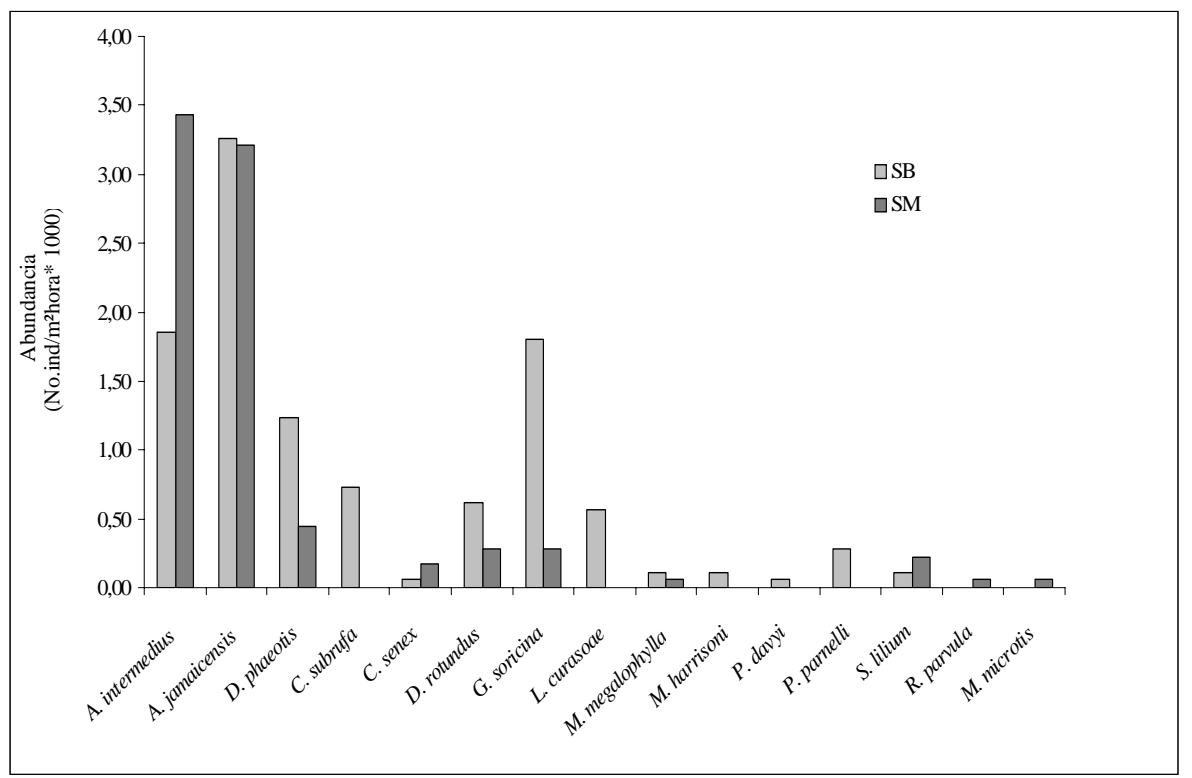

Figura 1. Abundancia relativa de las especies de murciélagos capturados en dos ambientes contrastantes en la Reserva de la Biosfera de Chamela Cuixmala, México. SB= selva baja, y $\mathrm{SM}=$ selva mediana. 

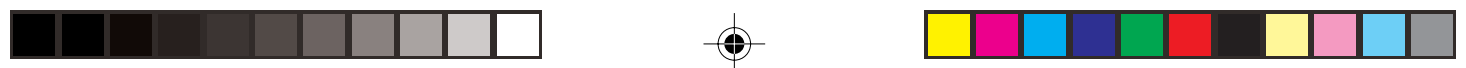

Diciembre 2001

a)

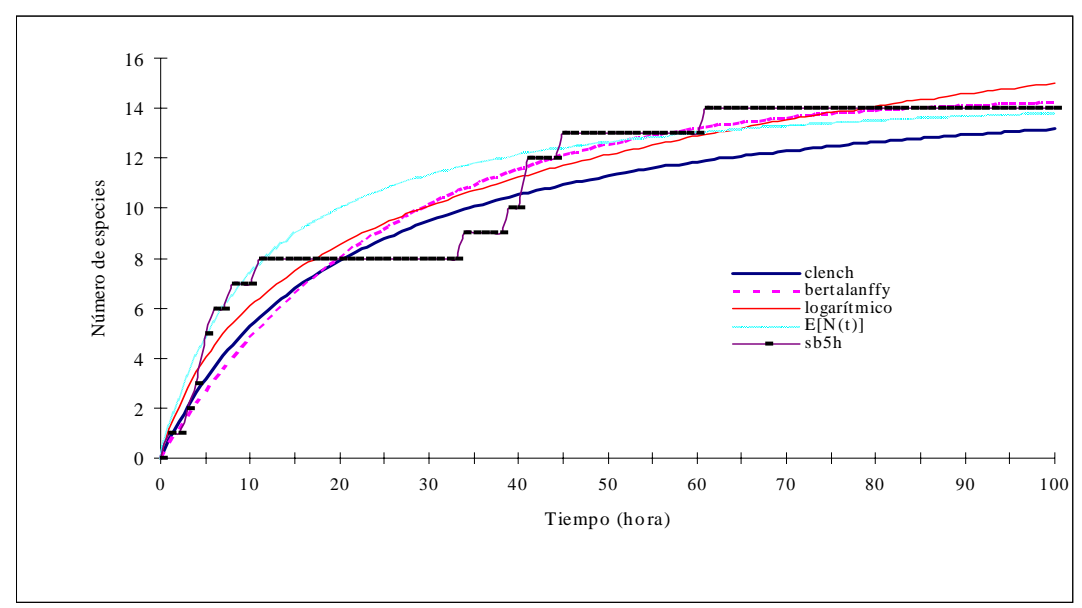

b)

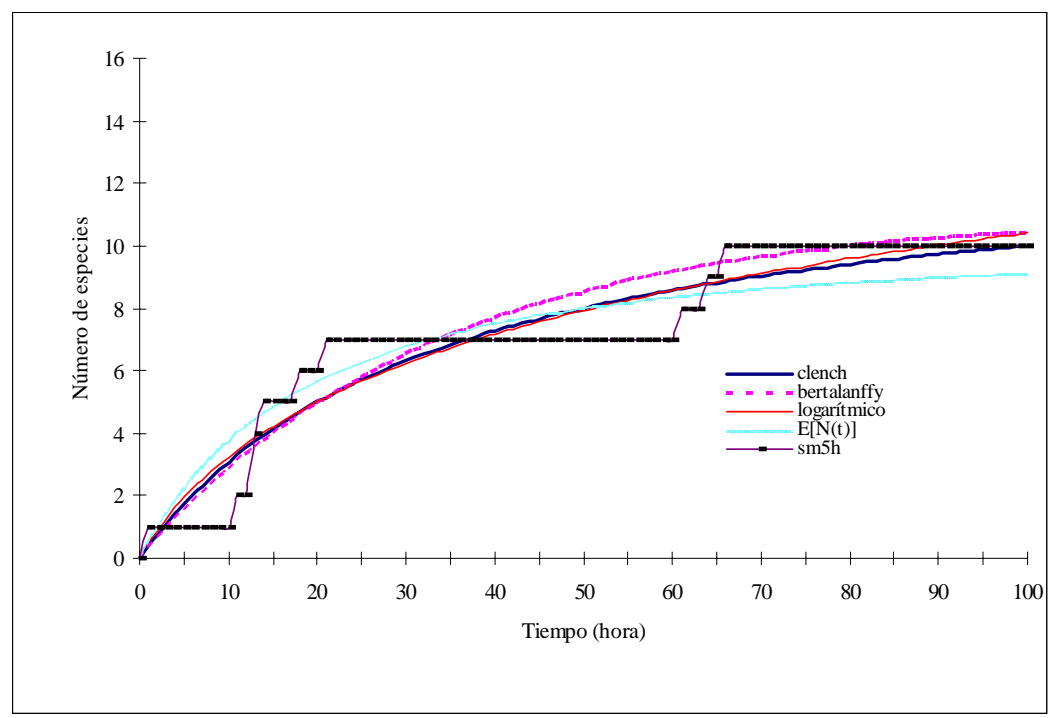

Figura 2 a y b. Curvas acumulativas de especies de murciélagos capturados en dos ambientes contrastantes en la Reserva de la Biosfera de Chamela Cuixmala, México con cuatro diferentes estimadores. $\mathrm{SB}=$ selva baja, y $\mathrm{SM}=$ selva mediana. 


\section{Variación Espacio-temporal}

Para comparar los dos sitios se utilizaron las especies compartidas. Al realizar un ANDEVA de tres vías encontramos que existen diferencias significativas entre los sitios, encontrándose una mayor abundancia (ind* ${ }^{2 *}$ red*hora; ANDEVA $\mathrm{F}_{\mathrm{gl}=1}=$ 4.670 $\mathrm{P}<0.04$ ) en la selva baja que en la selva mediana, además de diferencias entre las especies (ANDEVA $\mathrm{F}_{\mathrm{gl}=7}=39.16 \mathrm{P}<0.001$ ) y diferencias entre las temporadas (ANDEVA $\mathrm{F}_{\mathrm{gl}=5}=10.29 . \mathrm{P}<0.001$ ).

A. intermedius y A. jamaicensis presentaron una mayor abundancia que las otras seis especies. Además, D. phaeotis, y G. soricina presentaron una mayor abundancia que C.senex, S. lilium y M. megalophylla. Dentro de las temporadas encontramos que la temporada de lluvias del primer año, presentó una abundancia de murciélagos similar a la de la temporada de secas del primer año, y tuvo una mayor abundancia que las otros temporadas del primero y segundo años. En contraste, la temporada de secas del primer año tuvo una mayor abundancia de murciélagos que la de secas y transición del segundo año (Comparación de medias, prueba de Tukey $\mathrm{P}<$ $0.03)$.

En la selva seca se encontraron diferencias significativas en las abundancias de las especies (Andeva de dos vías, $\mathrm{F}_{\mathrm{gl}}=12.203 \mathrm{P}<0.001$ ) y no se encontraron diferencias temporales $\mathrm{F}_{\mathrm{gl} 17}=1.28 \mathrm{P}=0.30$ ). Artibeus jamaicensis presentó una mayor abundancia que $C$. senex, $M$. megalophylla, S. lilium y $D$. rotundus, siendo similar con $D$. phaeotis, A. intermedius y G. soricina. Además, G. soricina, A. intermedius y D. phaeotis fueron más abundantes que $C$. senex, $M$. megalophylla y S. lilium.

En la selva mediana encontramos diferencias en las abundancias entre especies $\left(\mathrm{F}_{\mathrm{gl} 7}=12.92 \mathrm{P}<0.001\right)$ y diferencias significativas entre las temporadas $\left(\mathrm{F}_{\mathrm{gl} 17}=5.81\right.$ $\mathrm{P}<0.001)$. Las especies A. jamaicensis y A. intermedius presentaron una mayor abundancia que las otras seis especies, y la abundancias de las dos especies fueron abundancias similares. La temporada de lluvias de 1992 presentó significativamente una mayor abundancia que todas las temporadas de 1993-94, y la de secas de 1992 presentó una mayor abundancia que la de post-lluvias de 1993-1994 (prueba de Tukey de comparación de medias $\mathrm{P}<0.04$ ).

Para analizar si hubo variación en la abundancia de los murciélagos por especie entre los dos sitios, a través de los dos años de muestreo y entre las temporadas, se consideraron a las 4 especies con mayor abundancia.

Evaluamos la variación temporal en cada una de las cuatro especies con mayor abundancia. Encontramos que A. intermedius no presentó diferencias estadísticamente significativas entre los sitios, los años y las temporadas de estudio (Andeva de tres 
vías $\mathrm{F}_{\mathrm{gl}=1,1,2}=0.89 ; 0.87$ y $4.35 \mathrm{P}>0.19$ ). Sin embargo, esta especie si presentó una mayor abundancia en el primer año (1992) en la selva mediana (Figura 3 a y b), causado por un pico en su abundancia en la temporada de lluvias de ese año.

Aunque Artibeus jamaicensis presentó una mayor abundancia en la selva mediana que en la selva baja y en la temporada de lluvias, tales diferencias fueron estadísticamente significativas $\left(\mathrm{F}_{\mathrm{gl}=2}=9.03 \mathrm{P}=0.10\right)$. Presentó dos picos de mayor abundancia, uno en abril y otro en octubre. Además fue la especie más abundante de la selva baja desde octubre de 1992 hasta junio de 1993.

En D. phaeotis se observó que su abundancia fue mayor tanto en el año de 1992 en comparación con 1993, y como en la selva baja en comparación con la selva mediana (Figura 3 a y b). Por temporadas presentó un patrón distinto de A. jamaicensis y A. intermedius, ya que su mayor abundancia se presentó en la temporada de secas (Figura 4 a y b).

G. soricina presentó abundancias similares en los dos años, pero en 1993 no fue capturada en la selva mediana. En las temporadas de lluvias y secas presentó abundancias similares, relativamente altas, y en la temporada de transición tuvo las densidades más bajas.

\section{DISCUSIÓN}

Las selvas secas de la costa de Jalisco experimentan una fuerte estacionalidad climática, con una pronunciada sequía anual, que influye fuertemente en las comunidades de plantas y animales. El efecto de la estacionalidad climática varía entre tipos de vegetación, y es más pronunciado en la selva baja cuando se compara con la selva mediana, debido a que esta última se localiza a lo largo de sitios con el manto freático superficial (Bullock et al., 1995). En consecuencia la diversidad y composición de especies varía temporal y espacialmente de manera dinámica (e.g. Ceballos, 1995).

Las respuestas de diferentes grupos de mamíferos a la estacionalidad de las selvas secas son diversas, y dependen en gran medida de su movilidad y fisiología. Algunas especies como las musarañas (Megasorex gigas) y el ratón Nyctomys sumichrasti se encuentran restringidas a la selva mediana, ya que no pueden sobrevivir la época de secas en la selva baja. Especies de poca movilidad como algunas especies de roedores (Osgoodomys banderanus y Peromyscus perfulvus) se dispersan a la selva baja durante la época de lluvias para retraerse a la selva mediana en la sequía. Otras especies como el ratón de abazones (Liomys pictus) pueden sobrevivir con una dieta de semillas, sin beber, obteniendo agua por medio de su metabolismo (Ceballos, 1989; Janzen y Wilson, 1993). Finalmente, especies de mayor tamaño o movilidad, 

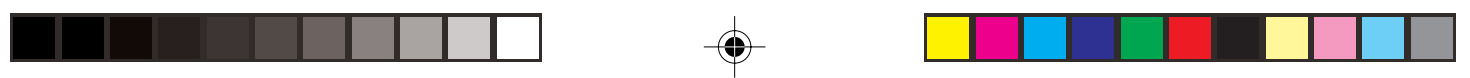

vol. 5

$\mathrm{SB})$

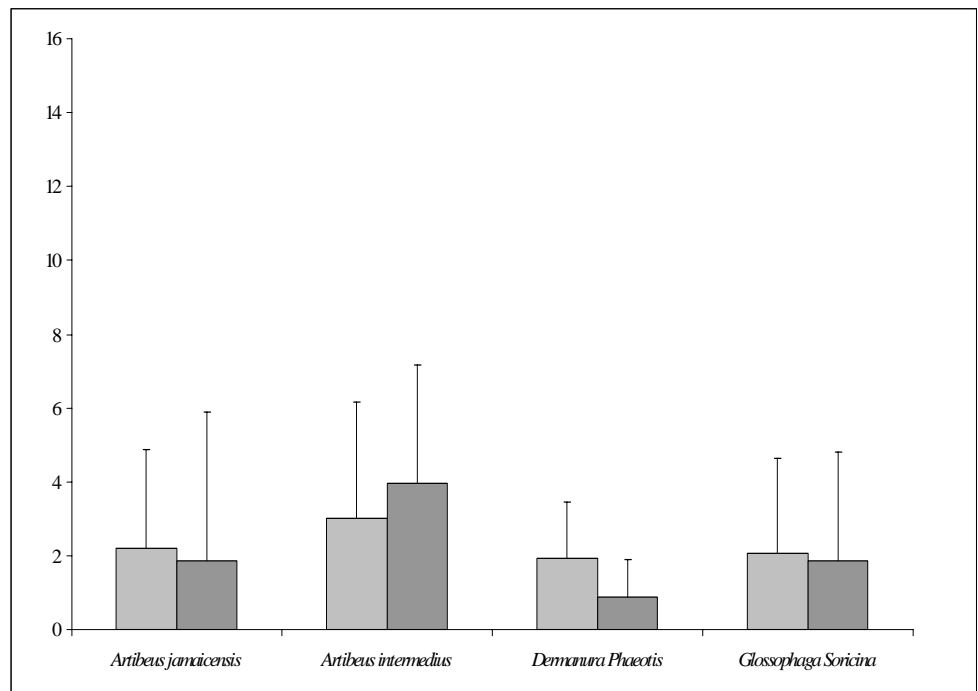

SM)

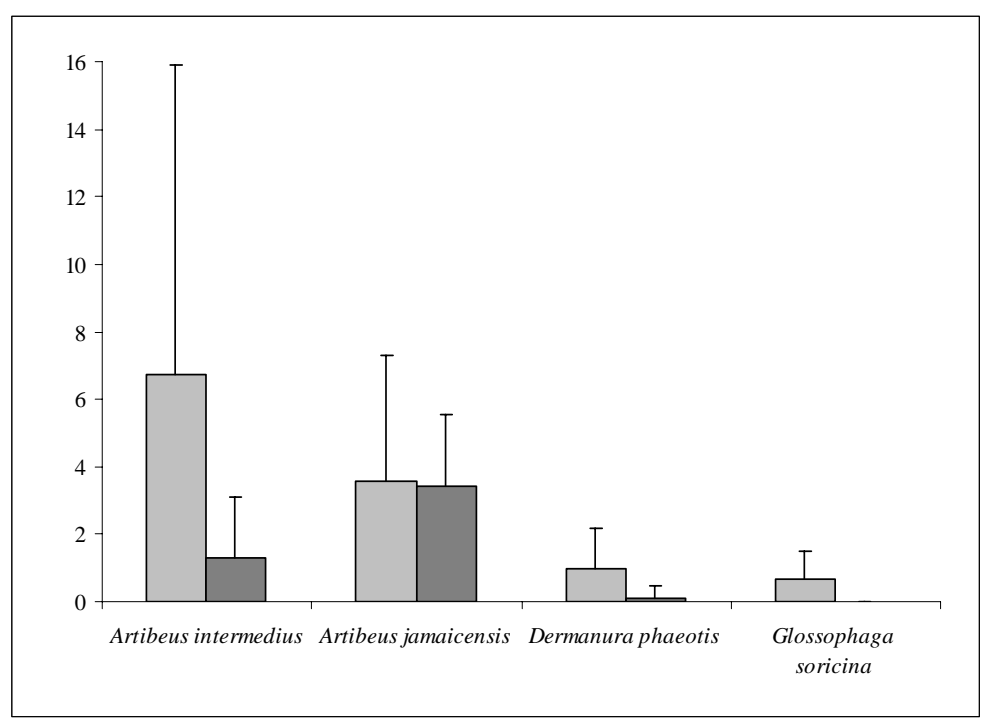

Figura 3a y b. Abundancia relativa de las cuatro especies de murciélagos comunes por año, en dos ambientes contrastantes en la Reserva de la Biosfera de Chamela Cuixmala, México. $\mathrm{SB}=$ selva baja, $\mathrm{y} \mathrm{SM}=$ selva mediana. 

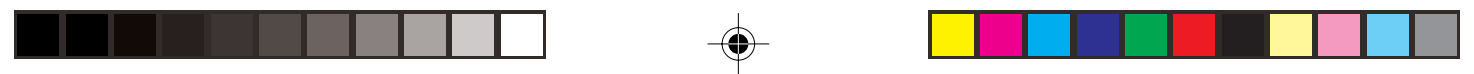

Diciembre 2001

a)

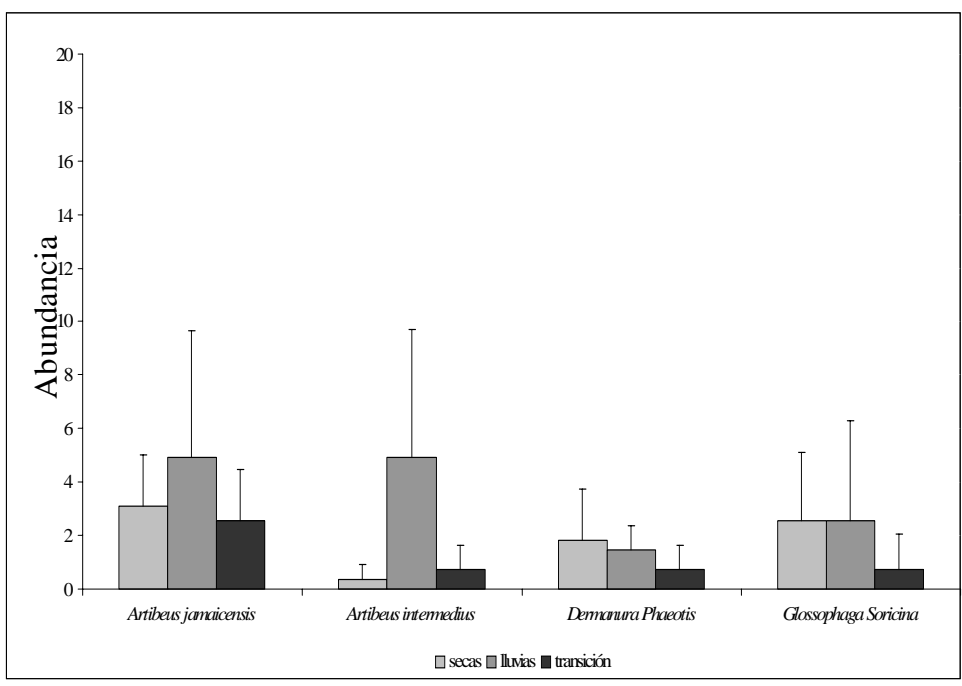

b)

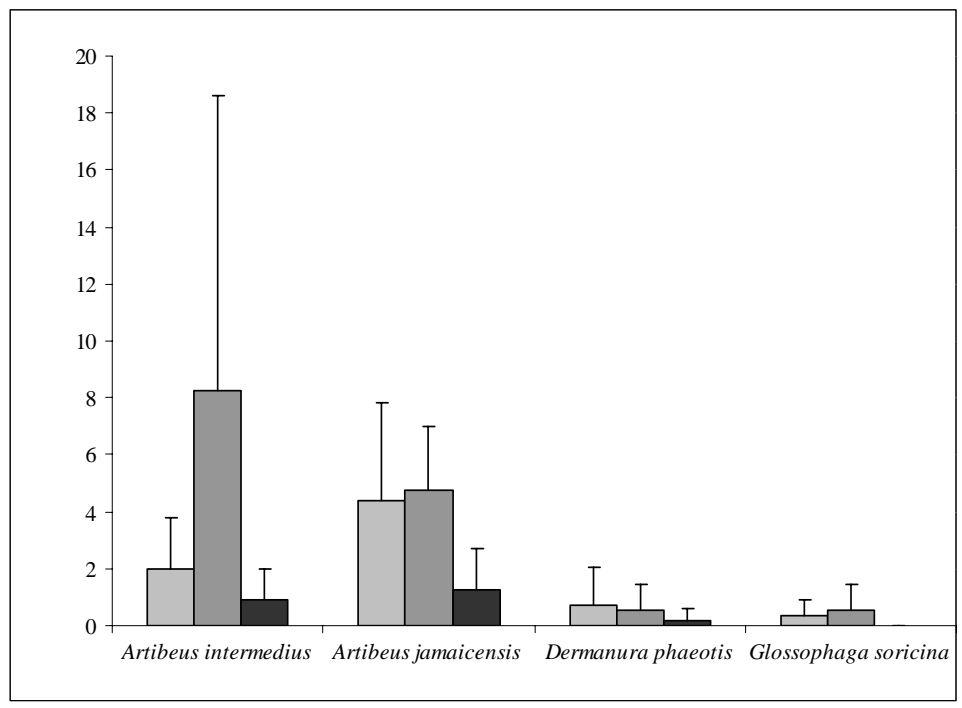

Figura 4 a y b. Abundancia relativa de las cuatro especies de murciélagos comunes por temporadas, en dos ambientes contrastantes en la Reserva de la Biosfera de Chamela Cuixmala, México. SB= selva baja, y SM= selva mediana. 
como venados (Odocoileus virginianus) pueden desplazarse diariamente varios kilómetros hasta las fuentes de agua, o bien migrar a regiones con mayor disponibilidad de alimentos (Janzen y Wilson, 1983; Valenzuela y Ceballos, 2000).

Nuestro estudio con la comunidad de murciélagos de las selvas secas ha mostrado que este grupo de mamíferos también exhibe respuestas diversas a la estacionalidad, que se traducen en una amplia variación temporal y espacial en la diversidad y composición de especies de las selvas baja y mediana.

El número de especies en la región de Chamela - Cuixmala es de 34 (Ceballos y Miranda, 2000); sin embargo, en este estudio sólo registramos 15 especies. Nosotros atribuimos las diferencias al muestro y a la especificidad de hábitat de algunas especies. En primer lugar, nuestro método de muestreo es adecuado para las especies que usan el sotobosque, como Artibeus jamaicensis, pero es ineficiente para las especies que vuelan en el dosel o arriba del dosel como los murciélagos molósidos (e.g. Molusus rufus), y son detectadas por otros métodos (Brosset y CharlesDominique, 1990; Kalko et al., 1996). En segundo lugar, varias de las especies que no se encontraron se localizan en ambientes diferentes como el murciélago pescados (Noctilio leporinus) que habita en manglares y el murciélago blanco (Diclidurus albus) que sólo se ha localizado en palmares (Ceballos y Miranda, 2000; Sánchez Hernández y Chávez, 1985).

La variación que documentamos entre las especies capturadas refleja patrones interesantes de abundancia relativa y variación temporal y espacial. La abundancia relativa de las especies conformó un patrón bien establecido en las comunidades de murciélagos tropicales, en donde pocas especies son muy abundantes y el resto son escasas (Fleming et al., 1972; Heithaus et al., 1975; Kalko et al., 1996; Medellín 1993; Medellín et al., 2000; Ramírez Pulido y Armella, 1987; Téllez-Girón, 1996). En Chamela - Cuixmala seis especies de murciélagos filostómidos fueron dominantes numéricamente; el resto variaron de comunes a escasas. Nuestros datos de la selva baja son muy parecidos a lo encontrado en la selva baja de Palo verde, Costa Rica, aunque las especies dominantes (Stoner 2001). La dominancia de los filostómidos, en especial de especies en los géneros Artibeus, Dermanura, Glossophaga y Desmodus es un indicador en algunas circunstancias de perturbaciones antrópicas (Fenton et al., 1992; Medellin et al., 2000). En Chamela - Cuixmala es posible que su dominancia sea un reflejo de una combinación de factores naturales y antropogénicos.

La variación temporal de las especies seguramente es una consecuencia, por lo menos parcialmente, de la estacionalidad climática (Ceballos, 1995; La Val y Finch, 1977). Las tres respuestas documentadas con mayor frecuencia son las migraciones, el uso estacional de ambientes y el cambio de dieta (Bonaccorso, 1979; Bonaccorso y Humphrey, 1984; Ceballos et al., 1997; 3Charles-Dominique, 1991; Dinerstein, 
1986; Fleming, 1988; Fleming y Heithaus, 1986; Fleming et al., 1972; Fleming, et al. 1993; Jansen y Wilson, 1983; Wilson, 1971).

En Chamela - Cuixmala desconocemos que tan extendidas son las migraciones regionales o geográficas. Hay evidencias indirectas de que algunas especies como Diclidurus albus se encuentran estacionalmente pero no se conoce donde se encuentran cuando desaparecen de la región (Ceballos y Miranda, 2000). El caso mejor documentado de movimientos a gran escala es el de Leptonycteris curasoae, que se desplaza de la costa de Jalisco hasta el sur de Estados Unidos en la época de reproducción (Ceballos et al., 1997). Es posible que otras especies como Pteronotus parnelli, Mormoops megalophilla y Natalus stramineus tambien realicen desplazamientos a distancias considerables, fenómeno que debe evaluarse.

El uso diferencial de la selva baja y la selva mediana (y otros ambientes como manglares) de varias especies es claro. La variación en la abundancia de varias de las especies dominantes entre la selva baja y la mediana refleja tales movimientos (Chávez, 1996). Estudios detallados con telemetría o marcadores de otro tipo permitirá determinar la magnitud de tales movimientos. Es interesante notar que sólo registramos a algunas especies muy especializadas como Musonycteris harrisoni en la selva seca, patrón que contradice en cierta manera la idea generalizada de que esta especie se encuentra casi exclusivamente en la selva mediana (Ceballos y Miranda, 2000). Información recabada en otras temporadas por nosotros nos ha permitido confirmar que esta especie se localiza también en la selva mediana en ciertas épocas del año; no tenemos suficientes datos, sin embargo, para determinar un patrón anual definido. Finalmente, la variación en la dieta de especies de murciélagos en la región de Chamela - Cuixmala es escasa, pero hay evidencias indirectas de que esto ocurre en especies como Carollia subrufa y Micronycteris microtis.

Es evidente que aún se desconocen aspectos básicos de la ecología de comunidades de los murciélagos de la región de Chamela - Cuixmala. Sin embargo, estudios como el nuestro están aportando información básica para entender los patrones y procesos fundamentales para mantener estas comunidades a largo plazo. El conocimiento generado tiende además interesantes implicaciones para la conservación, como la importancia de mantener las reducidas selvas medianas en la región, que son fundamentales en la dinámica espacio - temporal que mantienen estas comunidades.

\section{AGRADECIMIENTOS}

Queremos hacer patente nuestro sincero agradecimiento a la Fundación Ecológica de Cuixmala su apoyo para llevar a cabo este estudio. El financiamiento del estudio fue proporcionado por la UNAM. Andrés García apoyo en la logística. Finalmente, 
Yolanda Domínguez y Giselle Oliva nos ayudaron con la edición de la versión final del manuscrito.

\section{LITERATURA CITADA}

Amín, M. A. y R. A. Medellín. 1993. Un nuevo método para marcar murciélagos. Resumenes, Segundo Congreso Nacional de Mastozoología. Guadalajara, Jalisco, México.

Bonaccorso, F. J. 1979. Foraging and reproductive ecology in a Panamian bat community. Bulletin of the Florida State Museum, Biological Sciences, 24: 359-408.

Bonaccorso, F. J. y S. R. Humphrey. 1984. Fruit bat niche dynamics: their role in maintaining tropical forest diversity. Pp. 169-183, en: Proceedings of Tropical Rain-Forest. The Leeds Symposium (Chadwich A. C. y S. L. Sutton, eds). The Leeds Philosophical and Literary Society, Leeds, Gran Bretaña.

Brosset, A. y P. Charles-Dominique. 1990. The bats from French Guiana: a taxonomic, faunistic and ecological approach. Mammalia, 54:509-560.

Bullock, S. H. 1986. Climate of Chamela, Jalisco and trends in the south coastal region of Mexico. Arch. Met. Geaph. Biocl., Ser B, 36:297-316.

Bullock, S. H. y A. Solís-Magallanes. 1990. Phenology of canopy trees of a tropical deciduous forest in Mexico. Biotropica, 22:22-35.

Bullock, S. E. Medina y Mooney (eds). 1995. Seasonally Dry Tropical Forests. Cambridge University Press, Cambridge.

Ceballos, G. 1995. Vertebrate diversity, ecology, and conservation in NeoropicalDeciduous Forests. Pp. 195-220, en: Seasonally Dry Tropical Forests (Bullock, S.,E. Medina y H. Mooney, Eds.). Cambridge University Press, Cambridge.

Ceballos, G. y A. Miranda. 1986. Los mamíferos de Chamela, Jalisco. Instituto de Biología, UNAM, México, D.F.

Ceballos, G. y A. Miranda. 2000. Guía de campo de los mamíferos de la Costa de Jalisco, México. Fundación Ecológica de Cuixmala, A. C. - Universidad Nacional Autónoma de México, México D. F.

Ceballos, G. y A. García. 1995. Conserving neotropical biodiversity: the role of dry forest in México. Conservation Biology, 9:1349-1356.

Ceballos, G., T. H. Fleming, C. Chávez, C. y J. Nassar. 1997. Annual population cycle of Leptonycteris curasoae (Chiroptera, Phyllostomidae) at roost near Chamela, Jalisco, México. Journal of Mammalogy, 78:1220-1230.

Charles-Dominique, 1991. Feeding strategy and activity budget of the frugivorous bat Carollia perspicillata (Chiroptera: Phyllostomidae) in French Guiana. Journal of Tropical Ecology, 7:243-256.

Chávez, C. 1996. Análisis estadístico de la temporalidad de especies de murciélagos en Chamela, Jalisco, México. Trabajo Final, Especialización en Estadística Aplicada, Universidad Nacional Autónoma de México, México, D. F.

Dinerstein, 1986. Reproductive ecology of fruit bats and the seasonality of fruit production in a Costa Rican Cloud Forest. Biotropica, 18:307-318. 
Erkert, H. G. 1982. Ecological aspects of bat activity rhytms. Pp. 201-242, en: The ecology of bats (T. H. Kunz, editor). Plenum Press, Nueva York.

Fenton, M. B., L. Acharya, D. Audet, M. B. C. Hickey, C. Merriam, M. K. Obrist, D. M. Syme y B. Adkins. 1992. Phyllostomid bats (Chiroptera: phyllostomidae) as indicators of habitat disruption in the neotropics. Biotropica, 24:440-446.

Findley, J. S. 1993. Bats: A community perspective. Cambridge University Press, Cambridge.

Fleming, T. H. 1988. The short-tailed fruit bat. University of Chicago Press, Chicago.

Fleming, T. H. y E. R. Heithaus. 1986. Seasonal foranging behavior of Carollia perspicillata (Chiroptera: Phyllostomidae). Journal of Mammalogy, 67:660-671.

Fleming, T. H., E.T. Hooper y D. E. Wilson. 1972. Three Central American bat communities: structure, reproductive cycles, and movement patterns. Ecology, 53:555-569.

Fleming, T. H., R. A. Nuñez y L. S. Stenberg. 1993. Seasonal changes in the diets of migrant and non-migrant nectarivorous bats as revealed by carbon stable isotope analysis. Oecologia, 94:72-75.

Francis, C. M. 1990. Trophic structure of bat communities in the understory of lowland dipterocarp rainforest in Malasyia. Journal of Tropical Biology and Ecology, 13:293-349.

Heithaus, E. R. Fleming T. H. y P. W. Opler. 1975. Foraging patterns and resource utilization in seven species of bats in a seasonal tropical forest. Ecology, 56:841-854.

Iudica, C. A. 1994. Role of a bat community in the regeneration process of a forest after human disturbance in northwestern Argentina. Thesis of Masters of Arts, University of Florida, Gainesville.

Janzen, D. H. 1988. Tropical dry forest: the most endangered major tropical ecosystem. Pp. 130-137, en: Biodiversity. (E. O. Wilson, ed). National Academy Press, Washington, D. C.

Janzen, D.H. (ed). 1983. Costa Rican Natural History. University of Chicago Press, Chicago.

Jansen, D. H. y D. E. Wilson 1983. Mammals. Pp. 426-432, en: Costa Rican Natural History (D. H. Jansen, ed). Chicago University Press, Chicago.

Kalko, E. K. V. y C. O Handley, Jr. 2001. Neotropical bats in the canopy: diversity, community structure, and implications for conservation. Plant Ecology, 153:319-333.

Kalko, E. K. V, C. O. Handley y D. Handley. 1996. Organization, diversity and long-term dynamics of a Neotropical bat Community. Pp. 503-553, en. Long-term studies in vertebrate communities (Cody, M. y J. Smallwood, eds). Academic Press, Los Angeles.

LaVal, R. K. y H. S. Fitch. 1977. Structure, movements, and reproduction in three Costa Rican bat communities. Occasional Papers, Museum of Natural History, University of Kansas, 69:1-28.

Lott, E., S. Bullock y A. Solís-Magallanes. 1987. Floristic diversity and structure of upland and arroyo forest of coastal Jalisco. Biotropica, 19:228-235.

Martínez-Yrizar, A. 1995. Biomass distribution and primary productivity of tropical dry forests. Pp. 323-345, en: Seasonally Dry Forest, (S.H. Bullock, E. Medina y H. Mooney, eds.) Cambridge University Press, Cambridge, U. K.

McNab, B. K. 1971. The structure of tropical bat faunas. Ecology, 52:352-358.

Medellín, R. A. 1993. Estructura y diversidad de una comunidad de murciélagos en el trópico húmedo mexicano. Pp. 331-354, en: Avances en El Estudio de los Mamíferos de México 
(Medellín, R. A. y G. Ceballos, eds). Publicaciones Especiales 1, Asociación Mexicana de Mastozoología, A. C. México, D. F.

Medellín, R. A., Equihua, M. y M. A. Amín. 2000. Bat diversity and abundance as indicator of disturbance in Notropical rainforest. Conservation Biology, 14:1666-1675.

Nakamura, M. y F. Peraza. 1995. A model for species accumulation based on the multinomial distribution. Reporte técnico del Centro de Investigaciones en Matemáticas, D95-19. Guanajuato, México.

Ramírez-Pulido, J. y M. A. Armella. 1987. Activity patterns of Neotropical bats (Chiroptera: Phyllostomidae) in Guerrero, México. Southwestern Naturalist, 32:363-370.

Soberón, J. M. y J. E. Llorente. 1993. The use of accumulation functions for the prediction of species richness. Conservation Biology, 7:480-488.

Stoner, K. E. 2001. Differential habitat use and reproductive patterns of frugivorous bats in tropical dry forest of northwestern Costa Rica. Canadian Journal of Zoology, 79:16261633.

Téllez-Girón, M. G. 1996. Murciélagos de la Costa Chica de Guerrero. Tesis de Licenciatura, Universidad Nacional Autónoma de México, México D. F.

Wilson, D. E. 1971. Ecology of Myotis nigricans (Mammalia: Chiroptera) on Barro Colorado Island, Panamá, Canal Zone. Journal of Zoology, 163:1-1 